Original Research Paper

\title{
Monte Carlos Simulation Approach to Population Dynamics of Sickle Cell Anaemia
}

\author{
${ }^{1,2}$ Olumuyiwa O. Akanbi, ${ }^{2}$ Sunday O. Edeki and ${ }^{1,2}$ Olumuyiwa A. Agbolade \\ ${ }^{1}$ Department of Mathematics and Statistics, Federal Polytechnic Ilaro, Nigeria \\ ${ }^{2}$ Department of Mathematics, Covenant University, Ota, Nigeria
}

Article history

Received: 27-05-2016

Revised: $17-02-2017$

Accepted: 15-03-2017

Corresponding Author:

Sunday O. Edeki

Department of Mathematics,

Covenant University, Ota,

Nigeria

E-mail: soedeki@yahoo.com,

\begin{abstract}
Sickle Cell Anaemia (SCA) is a serious inherited blood disorder where the red blood cells, which carry oxygen around the body develop abnormally. The mathematical dynamics of the disease remain poorly understood, as such this paper investigates the mathematical inheritance pattern of the disease by the application of Monte Carlos simulation technique which is a complementary approach to physical simulation Smith's statistical package was used as random number generator in which the simulated birth from different mating indicates that SS has an average of $2.4 \%$ neonates, AS has $29.9 \%$ and AA has $67.7 \%$. We thus, conclude that eradication of SCA is not visible. However, curative measure of SCA remains paramount.
\end{abstract}

Keywords: Non-Gestation Female, Sickler, Carrier, Normal Simulation, Population Dynamics

\section{Introduction}

Sickle Cell Anaemia (SCA) is a genetic disease originating from the abnormal sickle Hemoglobin molecules (Hbs). In hypoxic conditions, the intracellular Hbs solution transitions into a polymerized state, resulting in a series of alterations in the cell membrane functions. Kaul et al. (1983) established that Suspensions of Sickle Red Blood Cells (SSRBCS) contain heterogeneous cell density groups which can be roughly divided into four fractions according to the intracellular Mean Corpuscular Hemoglobin Concentration (MCHC). Fractions I (SSI) and II (SS2) with moderate MCHC are mainly composed of reticulocytes and dislocytes respectively, with MCHC similar to healthy cells. On the other hand, fractions III (SS3) and IV (SS4) with high MCHC are mainly composed of rigid dislocytes and Irreversible Sickle Cells (ISC).

Individual genotype: AA, AS, SS, SC or AC differs amongst the world's population (Seeley et al., 1998). Of interest however, is the fact that genetic mechanism on morphogenetic traits is still not clearly understood as it is seen to occur with variable frequency in different populations and thus useful in evaluating and analyzing evolutionary forces and classification (Das, 2003). Meanwhile, marked inter-individual variability in genetic and non-genetic factors has been said to posses the ability to influence the disposition of many endobiotics and xenobiotic affecting health (Lamba et al., 2002).

Sickle Cell Anaemia which is a genetically transmitted disease is caused by a defective allele (mutant form) of the gene coding for a sub unit of the haemoglobinprotein (Akanbi et al., 2017). The Sickle haemoglobin tends to precipitate or "clump together" within the red blood cells after releasing its oxygen. If the clumping is extensive the red blood cell assumes an abnormal sickle shape. These sickle red blood cells plug the blood vessels thus preventing normal red blood cell passage and consequently depriving the tissue of needed oxygen.

Each person has two copies of the gene that determines whether that person has Sickle Cell Anaemia. If both copies are "normal alleles" then only normal haemoglobin is produced AA. If one of the two alleles is defective then that person has a mixture of normal and Sickle haemoglobin: A condition known as Sickle Cell trait "AS" (Carrier). If both alleles are defective, essentially only sickle haemoglobin is made and the person has Sickle Cell Anaemia "SS".

The first case of SCA was reported in 1910 on a Jamaican student in the USA (Sergeant, 1985). The term SCA is a term first used by Mason in 1922 to describe the homozygous state (Paule et al., 2011). In 1949, Neel illustrated that SCA was transmitted as a recessive gene ' $\mathrm{S}$ '. But it is well known by scientist now that the gene is neither dominant nor recessive but of intermediate penetrance.

The allele causing Sickle Cell Anaemia is found most often in people of African ancestry. It was traced to one family in Ghana (krobo people) in 1670 . The " $S$ " gene is found mainly where malaria is endemic (Neville and Panepinto, 2015). It also occurs in people of Mediterranean, Arab, East India, South and Central American ancestry. From the world population of about 7,058, 157,073 (Number of people officially 
counted) (USCB, 2013). 5\% of world population lives with SCD (Lamba et al., 2002). Mathematically, about $352,907,854$ people have haemoglobin disorder "officially counted" while every year 300,000 infants are born with SCD, including 200,000 cases in Africa (59th WHA, 2006). Nigeria of about 150 million population with growth rate of $3.2 \%$ has prevailing rate of 150,000 offspring per year. Nigeria by the virtue of her population ranks first as a SCA endemic country in Africa with annual infant deaths totally around 100,000, $8 \%$ of infant mortality (Akanbi, 2015).

In the recent years, simulation has made life more physical. Based on a simulated annealing procedure and experimental observations.

Mathematical models of heredity are largely based on one-locus, two allele genes population, where little or no attempt is made to consider the dynamics of the population by simulation (Monte Carlo simulation technique). Nevertheless, success approaches have been made on the simulation of related issues of Sickle Cell Anemia. Roughgarden (1979) performed computer simulations of genetic drift where five computer simulations of drift in a population of 8 individuals, beginning with an initial frequency of each allele of 0.5 . He concluded that either allele can be eliminated at a long term simulation.

Ting (2007) in a case study approach to teaching genetics demonstrated the diagnosis using simulated restricted analysis of DNA.

Paule et al. (2011) developed population Pharmacokinetic (PK)-Pharmacodynamics (PD) models for hydroxyurea in order to characterize the exposure efficiency relationship and their variability, compare two dosing regimes by simulations and developed some recommendation for monitoring the treatment.

Lei and Em-Karniada (2012) studied the rheology and dynamics of sickle Red Blood Cells (RBCS) suspension under constant shear and in a tube. The authors observed that no occlusion was observed in a straight tube under any condition unless an adhesive dynamics model was explicitly incorporated into simulations that partially trapped sickle RBCS which led to the full occlusion in some cases.

Lei and Em-Karniada (2013) probed the vasoocclusion phenomena in sickle cell anaemia via mesoscopic simulations. The entire simulation framework is based on Dissipative Particle Dynamics (DPD), including model for RBCs, leukocytes, plasma and the wall boundaries. Their simulations of individual SS-RBCs in shear flow validated the hypothesis of the importance of cell rigidity and morphology and elucidated the distinct behaviours of each individual cell group under adhesive conditions.

Sharoff (2015) in his pilot study integrated a genetic component into simulation to further enhance the perceived genetic knowledge of both students and medical practitioners.
Table 1. Percentage frequency of genotype

\begin{tabular}{ll}
\hline Genotype & Frequency \\
\hline AA & $69 \%$ \\
AS & $28 \%$ \\
SS & $3 \%$ \\
\hline
\end{tabular}

Table 2. Distribution properties of genotypes

\begin{tabular}{llll}
\hline Genotype & Probability & Cumulative probability & Tag-numbers \\
\hline AA & 0.69 & 0.69 & $0-68$ \\
AS & 0.28 & 0.97 & $69-96$ \\
SS & 0.03 & 1.00 & $97-$ \\
\hline
\end{tabular}

Table 1 and 2 show the genotypes, their associated frequencies and distributions properties.

From the recent literature available, the simulation of the transmission dynamics of sickle cell anaemia still stands a gap to be filled mathematically. In the search to fill up this gap, the authors in this present work will therefore, take a physical simulation and then later introduce simulation techniques referred to as Monte Carlos Simulation Techniques (MCST) to simulate the transmission dynamics of the disease (SCA).

\section{Methodology and Data Analysis}

The result of the physical simulation model created the basis for the data analysis.

The method to be employed in the analysis of the different genotypic groups (namely AA, AS, SS) is the Monte Carlos Simulation. In order to implement the technique the following procedures is of great interest.

The Monte Carlo simulation procedures can be summarized in the following six steps (Kalavathy, 2002; Walters et al., 1996):

- Clearly define the problem:

- Identify the objective of the problem

- Identify the main factors which have the greatest effect on the objectives of the problem

- $\quad$ Construct an appropriate model:

- Specify the variables and parameters of the model.

- State the conditions under which the experiment is to be performed

- Define the relationship between the variables and parameters

- Prepare the model for experimentation:

- Define the starting conditions for the simulation

- Specify the number of runs of simulations to be made

- Using Step 1 to 3, experiment with the model:

- Define a coding system that will correlate the factors defined in step 1 with the random number to be generated for the simulation

- Select a random number generator and create the random numbers to be used in the simulation

- Associate the generated random numbers with the factors identified in step 1 and coded step $4 a$

- $\quad$ Summarize and examine the results obtained in step 4

- Evaluate the results of the simulation 
Table 3. Random numbers and genotypes

\begin{tabular}{|c|c|c|c|c|c|c|c|c|c|c|c|c|c|c|}
\hline \multirow[b]{2}{*}{$\begin{array}{l}\text { Couple's } \\
\text { No. }\end{array}$} & \multicolumn{7}{|c|}{ Random numbers } & \multicolumn{7}{|c|}{ Genotype } \\
\hline & $\begin{array}{l}1^{\text {st }} \text { Gen } \\
/ \text { Trial }\end{array}$ & $\begin{array}{l}2^{\text {nd }} \text { Gen } \\
/ \text { Trial }\end{array}$ & $\begin{array}{l}3^{\text {rd }} \text { Gen } \\
/ \text { Trial }\end{array}$ & $\begin{array}{l}4^{\text {th }} \text { Gen } \\
/ \text { Trial }\end{array}$ & $\begin{array}{l}5^{\text {th }} \text { Gen } \\
/ \text { Trial }\end{array}$ & $\begin{array}{l}6^{\text {th }} \text { Gen } \\
/ \text { Trial }\end{array}$ & $\begin{array}{l}7^{\text {th }} \text { Gen } \\
/ \text { Trial }\end{array}$ & $\begin{array}{l}1^{\text {st }} \text { Gen } \\
/ \text { Trial }\end{array}$ & $\begin{array}{l}2^{\text {nd }} \text { Gen } \\
/ \text { Trial }\end{array}$ & $\begin{array}{l}3^{\text {rd }} \text { Gen } \\
/ \text { Trial }\end{array}$ & $\begin{array}{l}4^{\text {th }} \text { Gen } \\
/ \text { Trial }\end{array}$ & $\begin{array}{l}5^{\text {th }} \text { Gen } \\
/ \text { Trial }\end{array}$ & $\begin{array}{l}6^{\text {th }} \text { Gen } \\
/ \text { Trial }\end{array}$ & $\begin{array}{l}7^{\text {th }} \text { Gen } \\
/ \text { Trial }\end{array}$ \\
\hline 1. & 59 & 42 & 4 & 97 & 70 & 42 & 3 & $\mathrm{AA}$ & $\mathrm{AA}$ & $\mathrm{AA}$ & SS & $\mathrm{AS}$ & $\mathrm{AA}$ & $\mathrm{AA}$ \\
\hline 2. & 80 & 66 & 98 & 82 & 88 & 63 & 82 & AS & AA & SS & AS & AS & $\mathrm{AA}$ & AS \\
\hline 3. & 2 & 98 & 3 & 74 & 36 & 56 & 29 & AA & SS & AA & AS & AA & AA & AA \\
\hline 4. & 7 & 43 & 66 & 12 & 29 & 49 & 15 & $\mathrm{AA}$ & $\mathrm{AA}$ & $\mathrm{AA}$ & $\mathrm{AA}$ & $\mathrm{AA}$ & $\mathrm{AA}$ & $\mathrm{AA}$ \\
\hline 5. & 52 & 0 & 24 & 74 & 94 & 3 & 31 & $\mathrm{AA}$ & $\mathrm{AA}$ & AA & AS & AS & $\mathrm{AA}$ & $\mathrm{AA}$ \\
\hline 6. & 73 & 37 & 49 & 10 & 58 & 46 & 77 & AS & AA & AA & AA & AA & $\mathrm{AA}$ & $\mathrm{AS}$ \\
\hline 7. & 49 & 31 & 11 & 54 & 65 & 35 & 48 & $\mathrm{AA}$ & $\mathrm{AA}$ & $\mathrm{AA}$ & $\mathrm{AA}$ & $\mathrm{AA}$ & $\mathrm{AA}$ & $\mathrm{AA}$ \\
\hline 8. & 89 & 64 & 99 & 7 & 44 & 68 & 8 & AS & $\mathrm{AA}$ & SS & $\mathrm{AA}$ & $\mathrm{AA}$ & $\mathrm{AA}$ & $\mathrm{AA}$ \\
\hline 9. & 76 & 75 & 45 & 63 & 86 & 44 & 70 & AS & $\mathrm{AS}$ & $\mathrm{AA}$ & $\mathrm{AA}$ & AS & $\mathrm{AA}$ & $\mathrm{AS}$ \\
\hline 10. & 54 & 90 & 16 & 13 & 0 & 8 & 77 & $\mathrm{AA}$ & AS & $\mathrm{AA}$ & $\mathrm{AA}$ & $\mathrm{AA}$ & $\mathrm{AA}$ & AS \\
\hline 11. & 94 & 50 & 17 & 64 & 6 & 86 & 8 & $\mathrm{AS}$ & $\mathrm{AA}$ & $\mathrm{AA}$ & $\mathrm{AA}$ & $\mathrm{AA}$ & $\mathrm{AS}$ & $\mathrm{AA}$ \\
\hline 12. & 92 & 94 & 35 & 68 & 29 & 67 & 30 & AS & AS & $\mathrm{AA}$ & $\mathrm{AA}$ & $\mathrm{AA}$ & $\mathrm{AA}$ & $\mathrm{AA}$ \\
\hline 13. & 40 & 47 & 79 & 70 & 26 & 78 & 36 & AA & AA & $\mathrm{AS}$ & AS & AA & $\mathrm{AS}$ & $\mathrm{AA}$ \\
\hline 14. & 95 & 42 & 31 & 57 & 44 & 53 & 11 & $\mathrm{AS}$ & $\mathrm{AA}$ & AA & $\mathrm{AA}$ & AA & $\mathrm{AA}$ & $\mathrm{AA}$ \\
\hline 15. & 16 & 48 & 53 & 35 & 49 & 70 & 57 & $\mathrm{AA}$ & $\mathrm{AA}$ & $\mathrm{AA}$ & $\mathrm{AA}$ & $\mathrm{AA}$ & AS & $\mathrm{AA}$ \\
\hline 16. & 14 & 36 & 5 & 48 & 11 & 10 & 79 & $\mathrm{AA}$ & $\mathrm{AA}$ & $\mathrm{AA}$ & $\mathrm{AA}$ & $\mathrm{AA}$ & $\mathrm{AA}$ & AS \\
\hline 17. & 26 & 20 & 39 & 24 & 5 & 8 & 15 & $\mathrm{AA}$ & AA & AA & $\mathrm{AA}$ & AA & $\mathrm{AA}$ & $\mathrm{AA}$ \\
\hline 18. & 19 & 80 & 71 & 40 & 80 & 7 & 9 & $\mathrm{AA}$ & AS & $\mathrm{AS}$ & $\mathrm{AA}$ & AS & $\mathrm{AA}$ & $\mathrm{AS}$ \\
\hline 19. & 54 & 0 & 34 & 32 & 30 & 88 & 19 & $\mathrm{AA}$ & $\mathrm{AA}$ & $\mathrm{AA}$ & $\mathrm{AA}$ & $\mathrm{AA}$ & AS & $\mathrm{AA}$ \\
\hline 20. & 28 & 76 & 71 & 1 & 43 & 9 & 56 & AA & AS & AS & $\mathrm{AA}$ & AA & $\mathrm{AA}$ & $\mathrm{AA}$ \\
\hline 21. & 66 & 10 & 86 & 25 & 90 & 33 & 27 & $\mathrm{AA}$ & $\mathrm{AA}$ & AS & $\mathrm{AA}$ & AS & $\mathrm{AA}$ & $\mathrm{AA}$ \\
\hline 22. & 73 & 77 & 69 & 65 & 21 & 77 & 13 & AS & AS & AS & $\mathrm{AA}$ & $\mathrm{AA}$ & AS & $\mathrm{AA}$ \\
\hline 23. & 67 & 87 & 88 & 29 & 87 & 20 & 69 & $\mathrm{AA}$ & AS & AS & $\mathrm{AA}$ & AS & $\mathrm{AA}$ & $\mathrm{AS}$ \\
\hline 24. & 8 & 66 & 39 & 49 & 26 & 9 & 88 & $\mathrm{AA}$ & $\mathrm{AA}$ & $\mathrm{AA}$ & $\mathrm{AA}$ & $\mathrm{AA}$ & $\mathrm{AA}$ & AS \\
\hline 25 & 16 & 44 & 54 & 82 & 29 & 35 & 70 & $\mathrm{AA}$ & $\mathrm{AA}$ & AA & AS & AA & $\mathrm{AA}$ & AS \\
\hline 26. & 82 & 35 & 70 & 6 & 63 & 2 & 77 & AS & $\mathrm{AA}$ & AS & $\mathrm{AA}$ & $\mathrm{AA}$ & $\mathrm{AA}$ & AS \\
\hline 27. & 59 & 82 & 30 & 64 & 33 & 67 & 93 & $\mathrm{AA}$ & AS & AA & $\mathrm{AA}$ & AA & AA & $\mathrm{AS}$ \\
\hline 28. & 34 & 70 & 5 & 45 & 76 & 23 & 67 & AA & AS & AA & $\mathrm{AA}$ & AS & AA & $\mathrm{AA}$ \\
\hline 29. & 38 & 82 & 44 & 38 & 10 & 55 & 21 & $\mathrm{AA}$ & AS & AA & $\mathrm{AA}$ & AA & $\mathrm{AA}$ & $\mathrm{AA}$ \\
\hline 30. & 61 & 17 & 34 & 43 & 13 & 16 & 8 & $\mathrm{AA}$ & $\mathrm{AA}$ & $\mathrm{AA}$ & $\mathrm{AA}$ & $\mathrm{AA}$ & $\mathrm{AA}$ & $\mathrm{AA}$ \\
\hline 31. & 93 & 93 & 31 & 99 & 76 & 23 & 29 & AS & AS & AA & $\mathrm{SS}$ & AS & $\mathrm{AA}$ & $\mathrm{AA}$ \\
\hline 32. & 0 & 23 & 39 & 47 & 47 & 61 & 71 & AA & AA & AA & $\mathrm{AA}$ & AA & $\mathrm{AA}$ & $\mathrm{AS}$ \\
\hline 33. & 61 & 90 & 98 & 31 & 37 & 82 & 12 & $\mathrm{AA}$ & AS & SS & $\mathrm{AA}$ & $\mathrm{AA}$ & AS & $\mathrm{AA}$ \\
\hline 34. & 3 & 27 & 32 & 23 & 11 & 23 & 97 & $\mathrm{AA}$ & $\mathrm{AA}$ & $\mathrm{AA}$ & $\mathrm{AA}$ & $\mathrm{AA}$ & $\mathrm{AA}$ & AS \\
\hline 35. & 62 & 92 & 75 & 30 & 85 & 52 & 85 & $\mathrm{AA}$ & AS & AS & $\mathrm{AA}$ & AS & AA & AS \\
\hline 36. & 35 & 40 & 9 & 23 & 56 & 81 & 19 & $\mathrm{AA}$ & $\mathrm{AA}$ & $\mathrm{AA}$ & $\mathrm{AA}$ & $\mathrm{AA}$ & AS & $\mathrm{AA}$ \\
\hline 37. & 17 & 9 & 8 & 62 & 32 & 56 & 69 & AA & AA & AA & $\mathrm{AA}$ & AA & $\mathrm{AA}$ & $\mathrm{AS}$ \\
\hline 38. & 46 & 6 & 59 & 25 & 18 & 90 & 4 & $\mathrm{AA}$ & AA & AA & $\mathrm{AA}$ & AA & AS & AA \\
\hline 39. & 25 & 8 & 95 & 20 & 31 & 6 & 12 & $\mathrm{AA}$ & $\mathrm{AA}$ & AS & $\mathrm{AA}$ & $\mathrm{AA}$ & $\mathrm{AA}$ & AA \\
\hline 40 & 56 & 36 & 70 & 76 & 54 & 0 & 40 & $\mathrm{AA}$ & AA & AS & AS & AA & AA & $\mathrm{AA}$ \\
\hline 41. & 38 & 80 & 91 & 73 & 44 & 48 & 46 & $\mathrm{AA}$ & AS & AS & AS & $\mathrm{AA}$ & $\mathrm{AA}$ & $\mathrm{AA}$ \\
\hline 42. & 14 & 95 & 9 & 28 & 83 & 0 & 43 & $\mathrm{AA}$ & AS & $\mathrm{AA}$ & $\mathrm{AA}$ & AS & $\mathrm{AA}$ & $\mathrm{AA}$ \\
\hline 43. & 88 & 98 & 29 & 49 & 76 & 75 & 10 & $\mathrm{AS}$ & SS & AA & $\mathrm{AA}$ & AS & AS & $\mathrm{AA}$ \\
\hline 44. & 62 & 82 & 87 & 44 & 78 & 59 & 53 & $\mathrm{AA}$ & AS & AS & $\mathrm{AA}$ & $\mathrm{AS}$ & AA & $\mathrm{AA}$ \\
\hline 45 & 65 & 18 & 56 & 57 & 4 & 64 & 48 & $\mathrm{AA}$ & AA & AA & $\mathrm{AA}$ & AA & AA & $\mathrm{AA}$ \\
\hline 46. & 77 & 63 & 67 & 69 & 49 & 44 & 23 & $\mathrm{AS}$ & $\mathrm{AA}$ & AA & AS & AA & $\mathrm{AA}$ & $\mathrm{AA}$ \\
\hline 47. & 88 & 81 & 91 & 37 & 61 & 35 & 56 & $\mathrm{AS}$ & AS & $\mathrm{AS}$ & $\mathrm{AA}$ & AA & $\mathrm{AA}$ & $\mathrm{AA}$ \\
\hline 48. & 38 & 21 & 1 & 91 & 4 & 69 & 91 & $\mathrm{AA}$ & $\mathrm{AA}$ & $\mathrm{AA}$ & AS & $\mathrm{AA}$ & AS & AS \\
\hline 49. & 67 & 60 & 70 & 13 & 43 & 9 & 86 & $\mathrm{AA}$ & AA & AS & $\mathrm{AA}$ & AA & AA & $\mathrm{AS}$ \\
\hline 50. & 24 & 45 & 73 & 89 & 80 & 51 & 76 & AA & $\mathrm{AA}$ & AS & AS & AS & $\mathrm{AA}$ & $\mathrm{AS}$ \\
\hline 51. & 33 & 60 & 40 & 0 & 7 & 71 & 23 & $\mathrm{AA}$ & $\mathrm{AA}$ & $\mathrm{AA}$ & $\mathrm{AA}$ & $\mathrm{AA}$ & AS & $\mathrm{AA}$ \\
\hline 52. & 0 & 11 & 64 & 68 & 70 & 50 & 93 & $\mathrm{AA}$ & $\mathrm{AA}$ & $\mathrm{AA}$ & $\mathrm{AA}$ & AS & $\mathrm{AA}$ & AS \\
\hline 53. & 88 & 7 & 7 & 46 & 99 & 18 & 97 & $\mathrm{AS}$ & $\mathrm{AA}$ & AA & $\mathrm{AA}$ & SS & $\mathrm{AA}$ & SS \\
\hline 54. & 78 & 64 & 35 & 34 & 5 & 71 & 86 & AS & $\mathrm{AA}$ & $\mathrm{AA}$ & $\mathrm{AA}$ & $\mathrm{AA}$ & AS & AS \\
\hline 55. & 94 & 68 & 47 & 60 & 93 & 98 & 19 & AS & AA & AA & $\mathrm{AA}$ & $\mathrm{AS}$ & SS & AA \\
\hline 56. & 68 & 57 & 42 & 77 & 1 & 53 & 24 & $\mathrm{AA}$ & $\mathrm{AA}$ & $\mathrm{AA}$ & AS & $\mathrm{AA}$ & $\mathrm{AA}$ & $\mathrm{AA}$ \\
\hline 57. & 39 & 87 & 38 & 75 & 28 & 82 & 31 & AA & AS & AA & AS & AA & $\mathrm{AS}$ & AA \\
\hline 58. & 29 & 72 & 68 & 91 & 85 & 26 & 44 & $\mathrm{AA}$ & AS & AA & AS & $\mathrm{AS}$ & $\mathrm{AA}$ & AA \\
\hline 59. & 39 & 5 & 59 & 52 & 51 & 4 & 34 & AA & AA & AA & $\mathrm{AA}$ & AA & AA & AA \\
\hline 60. & 49 & 2 & 27 & 86 & 6 & 12 & 78 & $\mathrm{AA}$ & $\mathrm{AA}$ & $\mathrm{AA}$ & AS & $\mathrm{AA}$ & $\mathrm{AA}$ & AS \\
\hline 61. & 66 & 63 & 25 & 52 & 90 & 72 & 65 & $\mathrm{AA}$ & $\mathrm{AA}$ & AA & $\mathrm{AA}$ & $\mathrm{AS}$ & $\mathrm{AS}$ & AA \\
\hline 62. & 63 & 16 & 59 & 82 & 93 & 46 & 72 & AA & $\mathrm{AA}$ & AA & AS & AS & $\mathrm{AA}$ & AS \\
\hline 63. & 94 & 31 & 22 & 97 & 78 & 47 & 75 & $\mathrm{AS}$ & AA & AA & SS & AS & AA & AS \\
\hline
\end{tabular}




\begin{tabular}{|c|c|c|c|c|c|c|c|c|c|c|c|c|c|c|}
\hline 64. & 11 & 79 & 19 & 89 & 26 & 88 & 20 & $\mathrm{AA}$ & AS & $\mathrm{AA}$ & AS & $\mathrm{AA}$ & $\mathrm{AS}$ & $\mathrm{AA}$ \\
\hline 65. & 5 & 47 & 63 & 11 & 28 & 85 & 65 & $\mathrm{AA}$ & $\mathrm{AA}$ & AA & $\mathrm{AA}$ & $\mathrm{AA}$ & $\mathrm{AS}$ & AA \\
\hline 66. & 46 & 71 & 31 & 28 & 27 & 59 & 10 & $\mathrm{AA}$ & AS & AA & $\mathrm{AA}$ & $\mathrm{AA}$ & $\mathrm{AA}$ & $\mathrm{AA}$ \\
\hline 67. & 81 & 25 & 20 & 79 & 93 & 50 & 66 & AS & AA & AA & $\mathrm{AS}$ & AS & $\mathrm{AA}$ & $\mathrm{AA}$ \\
\hline 68. & 18 & 10 & 9 & 32 & 46 & 13 & 66 & $\mathrm{AA}$ & AA & AA & $\mathrm{AA}$ & AA & $\mathrm{AA}$ & AA \\
\hline 69. & 5 & 78 & 67 & 94 & 9 & 54 & 74 & $\mathrm{AA}$ & AS & AA & AS & $\mathrm{AA}$ & $\mathrm{AA}$ & AS \\
\hline 70 . & 5 & 3 & 8 & 69 & 12 & 39 & 97 & $\mathrm{AA}$ & $\mathrm{AA}$ & $\mathrm{AA}$ & $\mathrm{AS}$ & $\mathrm{AA}$ & $\mathrm{AA}$ & SS \\
\hline 71. & 27 & 9 & 71 & 91 & 76 & 39 & 39 & $\mathrm{AA}$ & AA & AS & AS & AS & $\mathrm{AA}$ & AA \\
\hline 72. & 94 & 75 & 41 & 96 & 80 & 37 & 86 & AS & AS & AA & $\mathrm{AS}$ & $\mathrm{AS}$ & $\mathrm{AA}$ & AS \\
\hline 73. & 42 & 54 & 69 & 14 & 64 & 42 & 27 & AA & AA & $\mathrm{AS}$ & AA & AA & $\mathrm{AA}$ & AA \\
\hline 74. & 58 & 37 & 74 & 72 & 85 & 77 & 15 & $\mathrm{AA}$ & $\mathrm{AA}$ & AS & AS & AS & AS & $\mathrm{AA}$ \\
\hline 75 . & 87 & 18 & 21 & 26 & 49 & 46 & 84 & AS & $\mathrm{AA}$ & AA & $\mathrm{AA}$ & $\mathrm{AA}$ & $\mathrm{AA}$ & AS \\
\hline 76. & 28 & 57 & 16 & 1 & 82 & 71 & 90 & $\mathrm{AA}$ & $\mathrm{AA}$ & AA & $\mathrm{AA}$ & $\mathrm{AS}$ & $\mathrm{AS}$ & AS \\
\hline 77. & 47 & 86 & 5 & 98 & 55 & 48 & 54 & $\mathrm{AA}$ & AS & $\mathrm{AA}$ & SS & $\mathrm{AA}$ & $\mathrm{AA}$ & $\mathrm{AA}$ \\
\hline 78. & 81 & 38 & 25 & 41 & 86 & 18 & 24 & AS & $\mathrm{AA}$ & $\mathrm{AA}$ & $\mathrm{AA}$ & AS & $\mathrm{AA}$ & $\mathrm{AA}$ \\
\hline 79. & 89 & 11 & 84 & 96 & 65 & 95 & 63 & AS & $\mathrm{AA}$ & AS & AS & $\mathrm{AA}$ & AS & AA \\
\hline 80. & 37 & 13 & 42 & 6 & 29 & 81 & 23 & $\mathrm{AA}$ & $\mathrm{AA}$ & AA & $\mathrm{AA}$ & $\mathrm{AA}$ & AS & $\mathrm{AA}$ \\
\hline 81. & 20 & 51 & 82 & 90 & 94 & 77 & 46 & $\mathrm{AA}$ & $\mathrm{AA}$ & $\mathrm{AS}$ & $\mathrm{AS}$ & AS & AS & $\mathrm{AA}$ \\
\hline 82. & 46 & 70 & 41 & 85 & 30 & 71 & 74 & $\mathrm{AA}$ & AS & AA & $\mathrm{AS}$ & $\mathrm{AA}$ & $\mathrm{AS}$ & AS \\
\hline 83. & 14 & 69 & 61 & 96 & 86 & 61 & 48 & $\mathrm{AA}$ & AS & $\mathrm{AA}$ & AS & AS & $\mathrm{AA}$ & AA \\
\hline 84. & 70 & 90 & 69 & 80 & 99 & 52 & 65 & AS & AS & AS & AS & SS & $\mathrm{AA}$ & $\mathrm{AA}$ \\
\hline 85. & 85 & 78 & 46 & 96 & 5 & 9 & 97 & AS & AS & $\mathrm{AA}$ & AS & $\mathrm{AA}$ & AS & SS \\
\hline 86. & 93 & 15 & 7 & 70 & 11 & 40 & 61 & AS & $\mathrm{AA}$ & AA & AS & $\mathrm{AA}$ & $\mathrm{AA}$ & $\mathrm{AA}$ \\
\hline 87. & 2 & 57 & 38 & 73 & 33 & 31 & 47 & $\mathrm{AA}$ & $\mathrm{AA}$ & $\mathrm{AA}$ & $\mathrm{AS}$ & $\mathrm{AA}$ & $\mathrm{AA}$ & $\mathrm{AA}$ \\
\hline 88. & 86 & 65 & 23 & 69 & 76 & 66 & 27 & AS & $\mathrm{AA}$ & AA & AS & AS & $\mathrm{AA}$ & $\mathrm{AA}$ \\
\hline 89. & 36 & 95 & 85 & 58 & 91 & 94 & 6 & $\mathrm{AA}$ & AS & AS & $\mathrm{AA}$ & AS & AS & $\mathrm{AA}$ \\
\hline 90. & 74 & 54 & 33 & 64 & 54 & 11 & 15 & AS & $\mathrm{AA}$ & AA & $\mathrm{AA}$ & $\mathrm{AA}$ & $\mathrm{AA}$ & $\mathrm{AA}$ \\
\hline 91. & 64 & 39 & 37 & 84 & 36 & 69 & 64 & $\mathrm{AA}$ & $\mathrm{AA}$ & $\mathrm{AA}$ & $\mathrm{AS}$ & $\mathrm{AA}$ & $\mathrm{AS}$ & $\mathrm{AA}$ \\
\hline 92. & 2 & 89 & 12 & 3 & 57 & 17 & 83 & $\mathrm{AA}$ & AS & $\mathrm{AA}$ & $\mathrm{AA}$ & $\mathrm{AA}$ & $\mathrm{AA}$ & AS \\
\hline 93. & 86 & 36 & 53 & 88 & 1 & 28 & 43 & $\mathrm{AS}$ & $\mathrm{AA}$ & AA & $\mathrm{AS}$ & $\mathrm{AA}$ & $\mathrm{AA}$ & AA \\
\hline 94. & 47 & 84 & 35 & 57 & 0 & 20 & 62 & $\mathrm{AA}$ & AS & $\mathrm{AA}$ & $\mathrm{AA}$ & $\mathrm{AA}$ & $\mathrm{AA}$ & $\mathrm{AA}$ \\
\hline 95. & 14 & 14 & 71 & 65 & 3 & 15 & 15 & $\mathrm{AA}$ & $\mathrm{AA}$ & AS & $\mathrm{AA}$ & $\mathrm{AA}$ & $\mathrm{AA}$ & $\mathrm{AA}$ \\
\hline 96. & 28 & 58 & 42 & 2 & 69 & 67 & 33 & $\mathrm{AA}$ & $\mathrm{AA}$ & AA & $\mathrm{AA}$ & AS & $\mathrm{AA}$ & AA \\
\hline 97. & 99 & 93 & 31 & 75 & 21 & 58 & 63 & SS & AS & $\mathrm{AA}$ & AS & $\mathrm{AA}$ & $\mathrm{AA}$ & AA \\
\hline 98. & 88 & 52 & 22 & 43 & 88 & 11 & 48 & $\mathrm{AS}$ & $\mathrm{AA}$ & $\mathrm{AA}$ & $\mathrm{AA}$ & AS & $\mathrm{AA}$ & $\mathrm{AA}$ \\
\hline 99. & 30 & 86 & 71 & 38 & 10 & 26 & 35 & $\mathrm{AA}$ & AS & AS & $\mathrm{AA}$ & $\mathrm{AA}$ & $\mathrm{AA}$ & $\mathrm{AA}$ \\
\hline 100. & 55 & 11 & 77 & 11 & 36 & 47 & 32 & $\mathrm{AA}$ & $\mathrm{AA}$ & AS & $\mathrm{AA}$ & $\mathrm{AA}$ & $\mathrm{AA}$ & $\mathrm{AA}$ \\
\hline 101. & 68 & 30 & 71 & 63 & 20 & 84 & 76 & $\mathrm{AA}$ & $\mathrm{AA}$ & $\mathrm{AS}$ & $\mathrm{AA}$ & $\mathrm{AA}$ & $\mathrm{AS}$ & AS \\
\hline 102. & 8 & 71 & 52 & 92 & 91 & 8 & 8 & $\mathrm{AA}$ & AS & $\mathrm{AA}$ & AS & AS & $\mathrm{AA}$ & $\mathrm{AA}$ \\
\hline 103. & 89 & 82 & 40 & 86 & 29 & 74 & 69 & $\mathrm{AS}$ & AS & $\mathrm{AA}$ & $\mathrm{AS}$ & $\mathrm{AA}$ & $\mathrm{AS}$ & AS \\
\hline 104. & 16 & 88 & 75 & 11 & 98 & 83 & 51 & AA & AS & $\mathrm{AS}$ & AA & SS & AS & AA \\
\hline 105. & 90 & 76 & 78 & 70 & 45 & 68 & 38 & AS & AS & AS & AS & $\mathrm{AA}$ & AS & $\mathrm{AA}$ \\
\hline 106. & 29 & 83 & 83 & 57 & 80 & 65 & 78 & $\mathrm{AA}$ & AS & AS & $\mathrm{AA}$ & AS & $\mathrm{AA}$ & AS \\
\hline 107. & 44 & 56 & 91 & 86 & 21 & 75 & 38 & $\mathrm{AA}$ & $\mathrm{AA}$ & AS & AS & $\mathrm{AA}$ & AS & $\mathrm{AA}$ \\
\hline 108. & 86 & 76 & 10 & 28 & 71 & 82 & 29 & $\mathrm{AS}$ & AS & $\mathrm{AA}$ & $\mathrm{AA}$ & AS & $\mathrm{AS}$ & $\mathrm{AA}$ \\
\hline 109. & 60 & 98 & 90 & 0 & 10 & 22 & 12 & $\mathrm{AA}$ & SS & AS & $\mathrm{AA}$ & $\mathrm{AA}$ & $\mathrm{AA}$ & $\mathrm{AA}$ \\
\hline 110. & 21 & 87 & 71 & 32 & 39 & 16 & 86 & $\mathrm{AA}$ & AS & AS & $\mathrm{AA}$ & $\mathrm{AA}$ & $\mathrm{AA}$ & AS \\
\hline 111. & 37 & 39 & 92 & 42 & 12 & 55 & 11 & $\mathrm{AA}$ & $\mathrm{AA}$ & AS & $\mathrm{AA}$ & $\mathrm{AA}$ & $\mathrm{AA}$ & $\mathrm{AA}$ \\
\hline 112. & 80 & 0 & 41 & 9 & 90 & 20 & 13 & $\mathrm{AS}$ & $\mathrm{AA}$ & $\mathrm{AA}$ & $\mathrm{AA}$ & AS & $\mathrm{AA}$ & $\mathrm{AA}$ \\
\hline 113. & 22 & 90 & 42 & 24 & 38 & 52 & 53 & $\mathrm{AA}$ & AS & AA & $\mathrm{AA}$ & $\mathrm{AA}$ & $\mathrm{AA}$ & AA \\
\hline 114. & 96 & 46 & 63 & 22 & 98 & 26 & 65 & AS & $\mathrm{AA}$ & $\mathrm{AA}$ & $\mathrm{AA}$ & SS & $\mathrm{AA}$ & $\mathrm{AA}$ \\
\hline 115. & 69 & 36 & 19 & 10 & 92 & 83 & 28 & AS & $\mathrm{AA}$ & $\mathrm{AA}$ & $\mathrm{AA}$ & AS & $\mathrm{AS}$ & $\mathrm{AA}$ \\
\hline 116. & 35 & 15 & 39 & 13 & 74 & 23 & 63 & $\mathrm{AA}$ & $\mathrm{AA}$ & $\mathrm{AA}$ & $\mathrm{AA}$ & AS & $\mathrm{AA}$ & $\mathrm{AA}$ \\
\hline 117. & 5 & 66 & 43 & 47 & 40 & 98 & 33 & $\mathrm{AA}$ & $\mathrm{AA}$ & AA & $\mathrm{AA}$ & $\mathrm{AA}$ & SS & $\mathrm{AA}$ \\
\hline 118. & 11 & 47 & 93 & 52 & 56 & 55 & 27 & $\mathrm{AA}$ & $\mathrm{AA}$ & $\mathrm{AS}$ & $\mathrm{AA}$ & $\mathrm{AA}$ & $\mathrm{AA}$ & $\mathrm{AA}$ \\
\hline 119. & 56 & 80 & 72 & 86 & 54 & 15 & 58 & $\mathrm{AA}$ & AS & AS & AS & $\mathrm{AA}$ & $\mathrm{AA}$ & $\mathrm{AA}$ \\
\hline 120. & 74 & 19 & 12 & 57 & 17 & 19 & 67 & $\mathrm{AS}$ & $\mathrm{AA}$ & $\mathrm{AA}$ & $\mathrm{AA}$ & $\mathrm{AA}$ & $\mathrm{AA}$ & $\mathrm{AA}$ \\
\hline 121. & 64 & 66 & 39 & 95 & 63 & 26 & 42 & AA & $\mathrm{AA}$ & $\mathrm{AA}$ & AS & $\mathrm{AA}$ & $\mathrm{AA}$ & AA \\
\hline 122. & 93 & 93 & 92 & 8 & 33 & 68 & 20 & $\mathrm{AS}$ & AS & $\mathrm{AS}$ & $\mathrm{AA}$ & $\mathrm{AA}$ & $\mathrm{AA}$ & $\mathrm{AA}$ \\
\hline 123. & 46 & 22 & 36 & 81 & 59 & 35 & 59 & AA & AA & $\mathrm{AA}$ & AS & $\mathrm{AA}$ & $\mathrm{AA}$ & AA \\
\hline 124. & 78 & 56 & 17 & 31 & 35 & 92 & 53 & $\mathrm{AS}$ & $\mathrm{AA}$ & $\mathrm{AA}$ & $\mathrm{AA}$ & $\mathrm{AA}$ & $\mathrm{AS}$ & $\mathrm{AA}$ \\
\hline 125. & 60 & 78 & 6 & 96 & 61 & 44 & 77 & AA & AS & $\mathrm{AA}$ & AS & $\mathrm{AA}$ & AA & AS \\
\hline 126. & 31 & 9 & 60 & 14 & 84 & 70 & 34 & $\mathrm{AA}$ & $\mathrm{AA}$ & AA & $\mathrm{AA}$ & AS & AS & $\mathrm{AA}$ \\
\hline 127. & 84 & 37 & 79 & 68 & 64 & 67 & 62 & $\mathrm{AS}$ & $\mathrm{AA}$ & $\mathrm{AS}$ & $\mathrm{AA}$ & $\mathrm{AA}$ & $\mathrm{AA}$ & $\mathrm{AA}$ \\
\hline 128. & 76 & 49 & 35 & 11 & 43 & 21 & 31 & AS & $\mathrm{AA}$ & $\mathrm{AA}$ & $\mathrm{AA}$ & $\mathrm{AA}$ & $\mathrm{AA}$ & AA \\
\hline 129. & 29 & 81 & 82 & 39 & 75 & 30 & 42 & $\mathrm{AA}$ & AS & $\mathrm{AS}$ & $\mathrm{AA}$ & AS & $\mathrm{AA}$ & $\mathrm{AA}$ \\
\hline 130. & 37 & 26 & 21 & 89 & 35 & 83 & 86 & $\mathrm{AA}$ & $\mathrm{AA}$ & AA & AS & $\mathrm{AA}$ & AS & AS \\
\hline 131. & 10 & 79 & 13 & 75 & 48 & 20 & 12 & $\mathrm{AA}$ & AS & $\mathrm{AA}$ & $\mathrm{AS}$ & $\mathrm{AA}$ & $\mathrm{AA}$ & $\mathrm{AA}$ \\
\hline
\end{tabular}


Olumuyiwa O. Akanbi et al. / American Journal of Applied Sciences 2017, 14 (3): 358.364 DOI: 10.3844/ajassp.2017.358.364

\begin{tabular}{|c|c|c|c|c|c|c|c|c|c|c|c|c|c|c|}
\hline 132. & 20 & 4 & 26 & 75 & 50 & 9 & 15 & $\mathrm{AA}$ & $\mathrm{AA}$ & $\mathrm{AA}$ & AS & $\mathrm{AA}$ & $\mathrm{AA}$ & $\mathrm{AA}$ \\
\hline 133. & 89 & 50 & 71 & 75 & 4 & 25 & 97 & AS & $\mathrm{AA}$ & AS & AS & $\mathrm{AA}$ & $\mathrm{AA}$ & SS \\
\hline 134. & 69 & 29 & 20 & 47 & 46 & 46 & 19 & AS & $\mathrm{AA}$ & $\mathrm{AA}$ & AA & $\mathrm{AA}$ & AA & $\mathrm{AA}$ \\
\hline 135. & 63 & 83 & 76 & 94 & 39 & 51 & 51 & AA & $\mathrm{AS}$ & AS & AS & $\mathrm{AA}$ & AA & $\mathrm{AA}$ \\
\hline 136. & 96 & 67 & 62 & 34 & 9 & 43 & 27 & AS & $\mathrm{AA}$ & AA & AA & $\mathrm{AA}$ & AA & $\mathrm{AA}$ \\
\hline 137. & 44 & 66 & 94 & 47 & 18 & 20 & 64 & AA & $\mathrm{AA}$ & AS & $\mathrm{AA}$ & $\mathrm{AA}$ & $\mathrm{AA}$ & $\mathrm{AA}$ \\
\hline 138. & 86 & 11 & 59 & 21 & 2 & 43 & 33 & AS & $\mathrm{AA}$ & AA & AA & $\mathrm{AA}$ & AA & $\mathrm{AA}$ \\
\hline 139. & 55 & 87 & 69 & 97 & 48 & 48 & 82 & AA & AS & AS & AS & $\mathrm{AA}$ & $\mathrm{AA}$ & AS \\
\hline 140. & 17 & 88 & 41 & 92 & 1 & 37 & 45 & $\mathrm{AA}$ & AS & $\mathrm{AA}$ & AS & $\mathrm{AA}$ & $\mathrm{AA}$ & $\mathrm{AA}$ \\
\hline 141. & 4 & 73 & 62 & 68 & 45 & 72 & 82 & AA & AS & $\mathrm{AA}$ & $\mathrm{AA}$ & $\mathrm{AA}$ & AS & AS \\
\hline 142. & 60 & 71 & 84 & 19 & 52 & 98 & 70 & $\mathrm{AA}$ & AS & AS & $\mathrm{AA}$ & $\mathrm{AA}$ & SS & AS \\
\hline 143. & 9 & 53 & 80 & 87 & 43 & 45 & 94 & AA & $\mathrm{AA}$ & AS & AS & $\mathrm{AA}$ & AA & AS \\
\hline 144. & 8 & 83 & 56 & 82 & 42 & 39 & 45 & $\mathrm{AA}$ & $\mathrm{AS}$ & $\mathrm{AA}$ & AS & $\mathrm{AA}$ & $\mathrm{AA}$ & $\mathrm{AA}$ \\
\hline 145. & 74 & 62 & 68 & 52 & 14 & 76 & 87 & AS & $\mathrm{AA}$ & AA & AA & $\mathrm{AA}$ & AS & AS \\
\hline 146. & 99 & 3 & 72 & 42 & 40 & 60 & 40 & SS & $\mathrm{AA}$ & AA & AA & $\mathrm{AA}$ & AA & $\mathrm{AA}$ \\
\hline 147. & 21 & 53 & 48 & 29 & 38 & 50 & 51 & AA & $\mathrm{AA}$ & AA & AA & $\mathrm{AA}$ & AA & $\mathrm{AA}$ \\
\hline 148. & 54 & 2 & 1 & 4 & 27 & 35 & 14 & AA & $\mathrm{AA}$ & $\mathrm{AA}$ & $\mathrm{AA}$ & $\mathrm{AA}$ & $\mathrm{AA}$ & $\mathrm{AA}$ \\
\hline 149. & 12 & 59 & 3 & 72 & 3 & 54 & 39 & AA & $\mathrm{AA}$ & $\mathrm{AA}$ & $\mathrm{AS}$ & $\mathrm{AA}$ & $\mathrm{AA}$ & $\mathrm{AA}$ \\
\hline 150 . & 66 & 60 & 86 & 86 & 25 & 5 & 85 & AA & $\mathrm{AA}$ & AS & AS & $\mathrm{AA}$ & AA & AS \\
\hline 151. & 88 & 68 & 25 & 27 & 22 & 39 & 41 & AS & $\mathrm{AA}$ & $\mathrm{AA}$ & AA & $\mathrm{AA}$ & $\mathrm{AA}$ & $\mathrm{AA}$ \\
\hline 152. & 66 & 17 & 16 & 63 & 49 & 57 & 56 & AA & $\mathrm{AA}$ & AA & AA & $\mathrm{AA}$ & AA & $\mathrm{AA}$ \\
\hline 153. & 29 & 75 & 25 & 96 & 98 & 85 & 55 & AA & AS & $\mathrm{AA}$ & AS & SS & AS & AS \\
\hline 154. & 82 & 77 & 87 & 83 & 4 & 15 & 99 & AS & AS & AS & AS & $\mathrm{AA}$ & $\mathrm{AA}$ & SS \\
\hline 155. & 7 & 45 & 24 & 1 & 16 & 89 & 96 & AA & $\mathrm{AA}$ & AA & AA & $\mathrm{AA}$ & AS & AS \\
\hline 156. & 38 & 73 & 24 & 4 & 71 & 6 & 85 & AA & $\mathrm{AS}$ & AA & AA & AS & AA & AS \\
\hline
\end{tabular}

Table 4. Result of birth from different mating

\begin{tabular}{|c|c|c|c|c|c|c|c|}
\hline \multirow[b]{2}{*}{ Genotype } & \multicolumn{7}{|l|}{ No of Birth } \\
\hline & 1st gen./trial & 2nd gen./trial & 3rd gen./trial & 4th gen./trial & 5th gen./trial & 6th gen./trial & 7th gen./trial \\
\hline $\mathrm{AA}$ & 107 & 98 & 106 & 97 & 110 & 114 & 107 \\
\hline AS & 47 & 55 & 47 & 55 & 41 & 39 & 43 \\
\hline SS & 2 & 3 & 3 & 4 & 5 & 3 & 6 \\
\hline
\end{tabular}

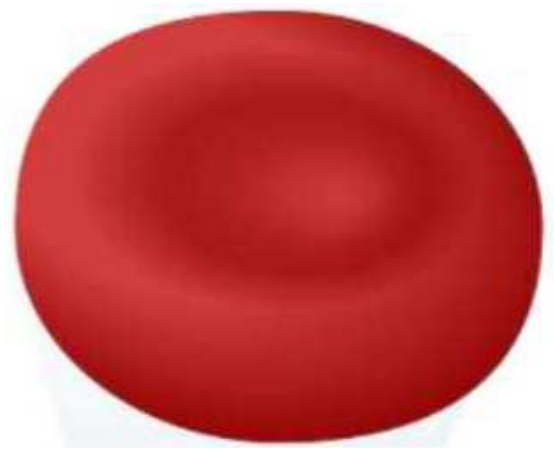

Fig. 1. Healthy-Normal Red Blood Cell (NRBC)

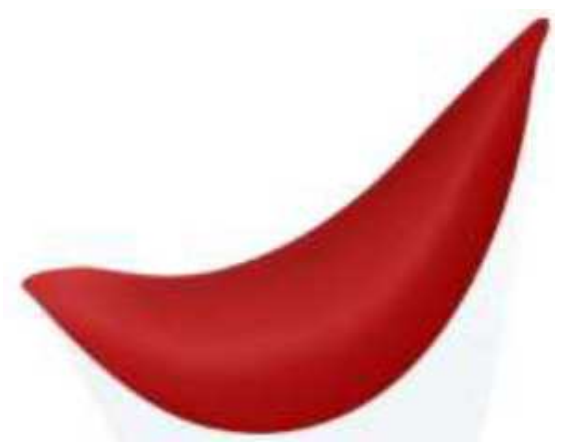

Fig. 2. Sickle Cell Amaenia- Sickle Red Blood Cell (SRBC)

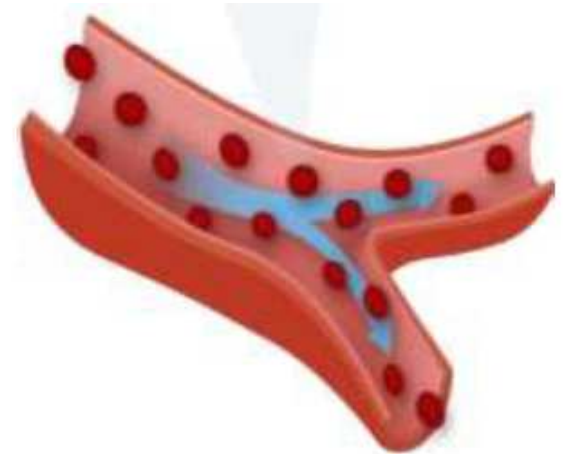

Fig. 3. Free blood flow-NRBC

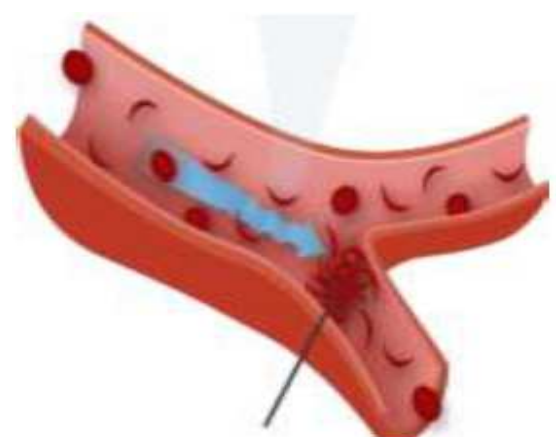

Fig. 4. Restricted blood flow-SRBC 


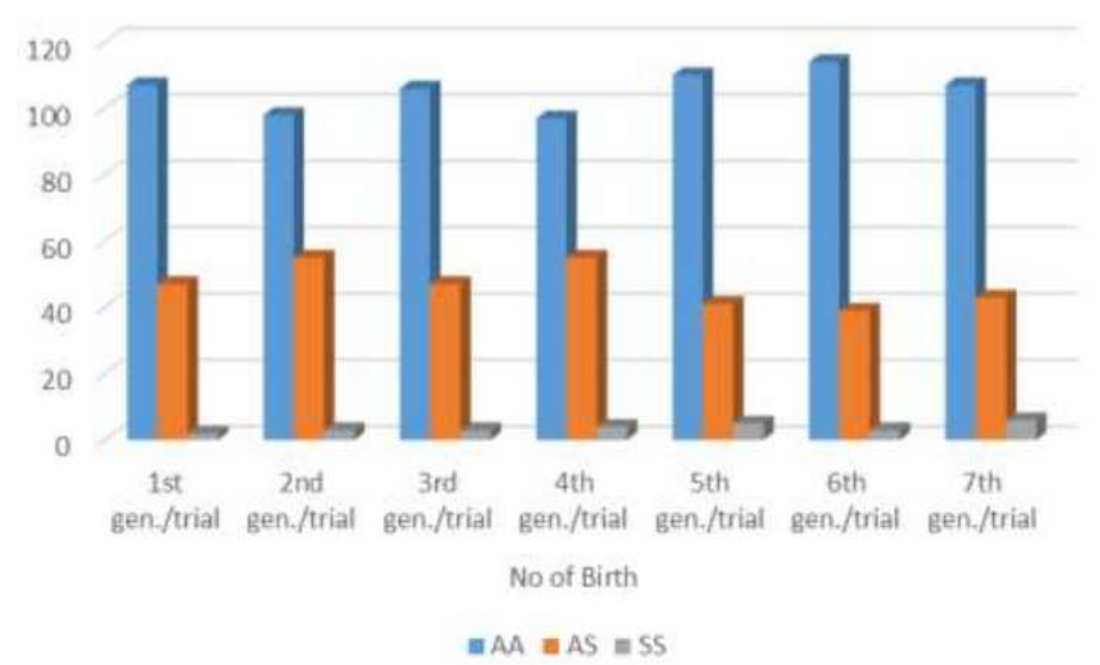

Fig. 5. Graphic for birth results from different mating

We present the following below: In Table 3, we show the simulation results; Table 4 shows the results of birth from different mating. In Fig. 1-4, we present graphics showing the blood cells of healthy normal red blood cell and that of sickle red blood cell with respect to free and restricted flow. While in Fig. 5, we have the graphical view for the birth results.

\section{Assumptions}

- The population consists of non gestating reproductive female with fertile male adults

- Immigration and emigration highly prohibited

- $\mathrm{AA}>\mathrm{AS}>\mathrm{SS}$

During the physical simulation, the birth of different genotypic group varied considerably with the distribution below (Akanbi, 2015).

We simulate for number of births of different genotypes namely AA, AS and SS. Considering 156 couples which is about $10 \%$ of the couples under consideration in physical simulation model (strictly under monogamy setting) (Akanbi, 2015). Hence, the next 156 neonates were simulated for seven generations/trials, while the random numbers were generated by SPP (Smith's Statistical Package).

From the distribution above, cumulative probability $\&$ Tag-numbers can be obtained as follows:

\section{Concluding Remark}

The investigation of the genetics of sickle cell trait via mathematical simulation reflects the importance of the concept of Monte Carlo technique in determining the population of sickle cell anaemia at any point in time from 156 births simulated for seven generations. The emergence of SS fluctuates with the highest occurrence of the 6 neotes being sickler. We observed that the social factor responsible for marriage that cannot be controlled mathematically is responsible for this fluctuations. Moreso, most marriages contracted in Nigeria and most developing countries were by accident. Hence, from simulated result the population of SS is not stable and there is great possibility for the population of SS to increase over time.

If genotype screening before marriage is duly imposed on the population, there will be abundant SS and AS classes in the population. Eventually, their relative activities will no longer be zero resulting to emergence of SS.

\section{Suggestion}

We need to face the fact that knowledge is still lacking on achieving a less expensive curative measures of sickle cell anaemia in Nigeria and on the entire tropical region where SCA is endemic. Though bone marrow transplant is a landmark measure which has proven effective in children but it is expensive. To achieve a remarkable growth, establishment of a body is paramount to scout and nurture innovative ideas and increase awareness of sickle cell anaemia.

However, the factors that are responsible for the unstable population of SCA can be examined mathematically for further research.

\section{Acknowledgement}

The authors are grateful to Covenant University for financial support. In addition, we thank the anonymous reviewer(s) for their constructive comments.

\section{Author's Contributions}

All authors contributed significantly in terms of idea and in preparation of this paper. 
Olumuyiwa O. Akanbi: Initiated the idea.

Sunday O. Edeki: Analyzed the data graphically and completed work.

Olumuyiwa A. Agbolade: Assisted in data collection.

\section{Ethics}

There is no ethical issues or conflict of interest regarding the publication of this paper.

\section{References}

Akanbi, O.O., 2015. Physical and monte carlos simulation of continuous time model of sickle cell anaemia. Proceedings of the 3rd National Conference of the School of Applied Science, (SAS' 15), Federal Polytechnic Ilaro.

Akanbi, O.O., S.O. Edeki and O.A. Agbolade, 2017. Continuous-time model and physical stimuliation of population dynamics of sickle cell anaemia. Int. J. Adv. Applied Sci.

Das, B., 2003. Sengupta: A note on some Morphogenetics variables among the Sonowal Kacharis of Assan. Anthropologist, 5: 211-212.

Kalavathy, S., 2002. Operations Research. 2nd Edn., Vikas Publishing House Pvt Limited, New Delhi, ISBN-10: 8125912754, pp: 506.

Kaul, D.K., M.E. Fabry, P. Windisch, S. Baez and R.I. Napel, 1983. Erythrocytes in sickle cell anamia are heterogeneous in their rheological hemodynamic characteristics. J. Clin. Invest, 72: 22-31.

Lamba, J.K., Y.S. Lin, K. Thummel, A. Daly and P.B. Watkins et al., 2002. Common allelic variants of cytochrome P4503A4 and their prevalence in different populations. Pharmacogenetics, 12: 121-132. PMID: 11875366

Lei, H. and G. Em-Karniadakis, 2012. Quantifying the rheological and hemodynamic characterisitcs of sickle cell Anaemia. Biophys. J., 102: 185-194.
Lei, H. and G. Em-Karniadakis, 2013. Probing vasoocclusion phenomena in sickle cell anaemia via mesoscopic simulations. Proc. Nat. Acad. Sci. USA, 110: 11326-11330.

Neville, A. and J.A. Panepinto, 2015. Pharmacotherapy of Sickle Cell Disease in Children. Curr. Pharm. Des., 21: 5660-56678. PMID: 26517528

Paule, I., H. Sassi, A. Habibi, K.P. Pham and D. Bachir et al., 2011. Population pharmacokinetics and pharmacodynamics of hydroxyurea in sickle cell anemia patients, a basis for optimizing the dosing regimen. Orphanet J. Rare Dis., 6: 30-30.

DOI: $10.1186 / 1750-1172-6-30$

Roughgarden, J., 1979. Theory of Population Heretics and Evolutionary Ecology: An Introduction. 1st Edn., Macmillan, New York.

Seeley, R.R., T.D. Stephens and P. Tate, 1998. Anatony and Physiology. 4th Edn., The McGraw Hill Companies, Inc. USA.

Sergeant, G.R., 1985. Sickle Cell Disease. 1st Edn., Oxford University Press, London, pp: 25.

Sharoff, L., 2015. Enhancing sickle cell Anaemiagenetic understanding through simulations: A descriptive pilot study. J. Nurs. Educ. Pract.

Ting, J., 2007. Chowing: A case study approach to teaching high school genetics. University Washington.

USCB, 2013. U.S census bureau.

Walters, M.C., M. Patience, W. Leisenring, J.R. Eckman and J.P. Scott et al., 1996. Bone marrow transplantation for sickle cell disease. N Engl. J. Med., 335: 369-376. PMID: 8663884

WHA, 2006. Fifty-ninth world health assembly provisional agenda item. 